\title{
Computation Time Analysis of the Magnetic Gear Analytical Model
}

\author{
M. Desvaux ${ }^{1}$, B. Traullé ${ }^{1}$, R. Le Goff Latimier ${ }^{1}$, S. Sire ${ }^{2}$, B. Multon ${ }^{1}$ and H. Ben Ahmed ${ }^{1}$ \\ ${ }^{1}$ SATIE, ENS Rennes, Université Bretagne Loire, CNRS, 35170 Bruz \\ ${ }^{2}$ University of Brest, FRE CNRS 3744, IRDL, 29238 Brest
}

\begin{abstract}
This article focuses on the computation time and precision of a linear 2D magnetic gear analytical model. Two main models of magnetic gears are studied: the first with an infinite relative permeability of yokes, and the second with a finite relative permeability of yokes. These models are based on the subdomain resolution of Laplace and Poisson equations. To accurately compute the magnetic field distribution, it is necessary to take into account certain harmonics of the various rings and other system harmonics due to modulation. Global system harmonics, which increase the value of computation time, must also be taken into account. If the magnetic gear has a high pole number, then computation time increases even more and no longer allows for system optimization. This article proposes to compute magnetic field distribution using different harmonic selection methods in order to significantly reduce the computation time for the magnetic torque without any loss of accuracy.
\end{abstract}

Index Terms - Analytical model, electromagnetic torque, harmonics, magnetic gear, optimization, precision, computation time, torque ripple, yoke.

\section{NOMENCLATURE}

$A^{(k)}$ : Magnetic vector potential of region $\mathrm{k}$

$B_{r}^{(k)}:$ Radial flux density in region $\mathrm{k}[\mathrm{T}]$

$B_{\alpha}^{(k)}$ : Tangential flux density in region $\mathrm{k}[\mathrm{T}]$

$C_{f}$ : Cogging torque factor

$G_{m}:$ Gear ratio

$k$ : Index of the magnetic gear region

$L_{z}$ : Magnetic length of the magnetic gear [m]

$M^{(k)}$ : Radial magnetization distribution of region $\mathrm{k}$

$N_{Q}$ : Number of harmonics taken into account in the air space

between pole pieces (region III)

$N$ : Number of harmonics taken into account in the other

regions (regions $\mathrm{X}, \mathrm{I}, \mathrm{II}, \mathrm{IV}, \mathrm{V}$ and $\mathrm{VI}$ )

$p_{\text {int }}:$ Number of internal ring pole pairs

$p_{\text {ext }}:$ Number of external ring pole pairs

$Q$ : Number of ferromagnetic pole pieces

$r$ : Cylindrical coordinate of the radius [m]

$R_{M}^{(k)}$ : External radius of region $\mathrm{k}[\mathrm{m}]$

$R_{m}^{(k)}$ : Internal radius of region $\mathrm{k}[\mathrm{m}]$

$T^{(k)}$ : Magnetic torque computed on region $\mathrm{k}[\mathrm{Nm}]$

$Z_{i, j}$ : Matrix system of model $i$ and harmonic selection method

$j$ with $i=1,2$ and $j=A, B, C, D$

$\alpha$ : Cylindrical coordinate of the angle [rad]

$\beta$ : Slot opening angle

$\mu_{0}$ : Relative permeability

$\mu_{k}$ : Relative permeability of region $\mathrm{k}$

$\omega_{\text {int } / 0}$ : Rotational speed of the internal ring [rad/s]

$\omega_{\text {ext } / 0}$ : Rotational speed of the external ring [rad/s]

$\omega_{Q / 0}:$ Rotational speed of the pole piece ring $[\mathrm{rad} / \mathrm{s}]$

$\delta_{\text {int }}:$ Internal air gap $[\mathrm{mm}]$

$\delta_{\text {ext }}:$ External air gap [mm]

Manuscript received Month XX, 2016; revised Month XX, 2016 and Month XX, 2016; accepted month XX, 2016. Date of publication Month XX, 2016; date of current version Month XX, 2016. Corresponding author: M. Desvaux (e-mail: melaine.desvaux@ens-rennes.fr).

Color versions of one or more of the figures in this paper are available online at: http://ieeexplore.ieee.org.

Digital Object Identifier (inserted by IEEE).

\section{INTRODUCTION}

In many drive chains, such as wind turbine drives [1]-[2], mechanical gearbox failure and maintenance increase operating costs, particularly in offshore locations. To improve drive train reliability, one potentially attractive solution consists of replacing the mechanical gearboxes by a magnetic gear [3]. The topology of such a magnetic gear with a high torque density (Fig. 1) was proposed in [4] and has been the subject of several studies [5]-[6]. The benefit of a magnetic gear over mechanical gearboxes is greater for high-torque application [7]. In this case, high pole numbers would be required. Moreover, cancellation of the ripple torque would necessitate the use of combinations of numbers of poles and slots with minimum magnetic symmetry [8], which leads to a very high computation time.

To demonstrate an economic improvement over mechanical gearboxes, a magnetic gear must be highly optimized with respect to both mechanical and magnetic criteria. Such an optimization would require a quick computation of the magnetic field distribution (on the order of one second) [9]. Then, an analytical magnetic field distribution model has been proposed in [10]. This model unfortunately does not allow for a quick computation of the magnetic field distribution like in

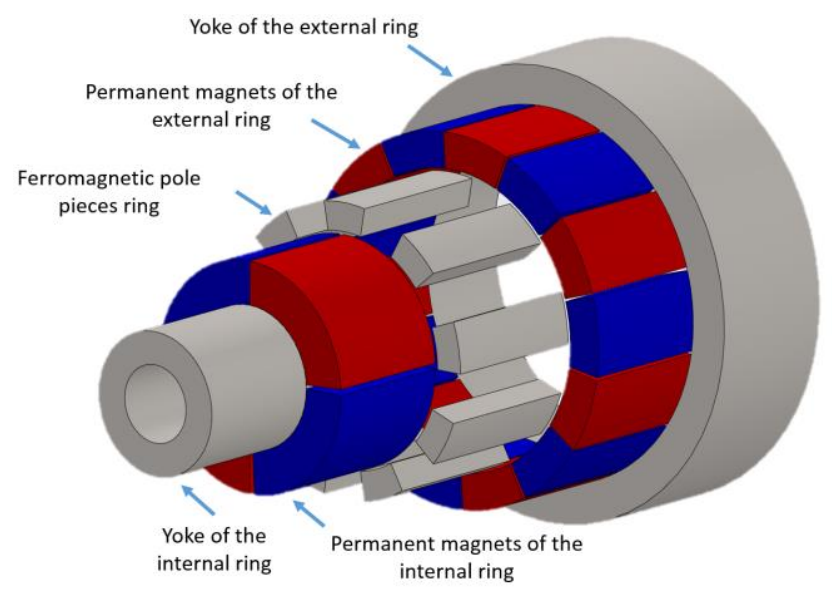

Fig. 1: Magnetic gear architecture proposed by [4] in an exploded-view drawing with low pole numbers (i.e. $p_{\text {int }}=2, p_{\text {ext }}=7$, and $Q=9$ ). 
[11] due to the fact that the magnetic gears with high pole numbers yet without torque ripple do not display magnetic symmetry [5] and [8]. It then becomes necessary to find another method capable of computing magnetic field distribution.

For a global magnetic gear optimization that contains a magnetic part optimization and a structural part optimization, it could be interesting to include the yokes of the permanent magnet rings in the optimization procedure to minimize magnetic part mass and material costs. This paper therefore seeks to provide an analytical model that includes yokes with finite relative permeability while proposing a harmonic selection method capable of reducing computation time without lowering precision for high pole number magnetic gears.

\section{PROBLEM DEFINITION}

\section{A. Magnetic gear behavior}

The magnetic gear [3] shown in Figure 1 is composed of three magnetic parts:

- An internal ring with $p_{\text {int }}$ pole pairs of permanent magnets and a ferromagnetic yoke,

- An external ring with $p_{\text {ext }}$ pole pairs of permanent magnets and a ferromagnetic yoke,

- A ring with Q ferromagnetic poles between the two permanent magnet rings (an example is shown in Fig. 1 with low pole numbers, to improve readability: $p_{\text {int }}=2$, $p_{\text {ext }}=7$, and $Q=9$ ).

The three ring pole numbers must respect the relation (1) [5]. It is then possible to define the ratio $\lambda$ (2) and the Willis relation for a magnetic gear (3) like a planetary gear [12], where $\omega_{\text {int } / 0}$, $\omega_{\text {ext } / 0}$ and $\omega_{Q / 0}$ are the rotational speeds of the internal ring, the external ring and the pole piece ring, respectively. As a function of the fixed ring, the gear ratio $G_{m}$ is given by (4).

$$
\begin{gathered}
p_{\text {int }}+p_{\text {ext }}=Q \\
\lambda=\frac{\omega_{\text {int } / 0}-\omega_{Q / 0}}{\omega_{\text {ext } / 0}-\omega_{Q / 0}}=-\frac{p_{\text {ext }}}{p_{\text {int }}} \\
\omega_{\text {int } / 0}-\lambda \cdot \omega_{\text {ext } / 0}+(\lambda-1) \cdot \omega_{Q / 0}=0 \\
\left\{\begin{array}{c}
\omega_{\text {int } / 0}=0 \rightarrow G_{m}=\frac{\omega_{\text {ext } / 0}}{\omega_{Q / 0}}=\frac{(\lambda-1)}{\lambda} \\
\omega_{Q / 0}=0 \rightarrow G_{m}=\frac{\omega_{\text {int } / 0}}{\omega_{\text {ext } / 0}}=\lambda \\
\omega_{\text {ext } / 0}=0 \rightarrow G_{m}=\frac{\omega_{\text {int } / 0}}{\omega_{Q / 0}}=-(\lambda-1)
\end{array}\right.
\end{gathered}
$$

To minimize the torque ripple, a cogging torque factor $C_{f}$ has been defined in [5], which represents the number of system symmetries. It is thus possible to impose the relation (5) to ensure a minimum torque ripple, where $N_{c}\left(2 . p_{\text {int }}, Q\right)$ is the smallest common multiple between 2. $p_{\text {int }}$ and $Q$. This relation also minimizes the number of magnetic symmetries.

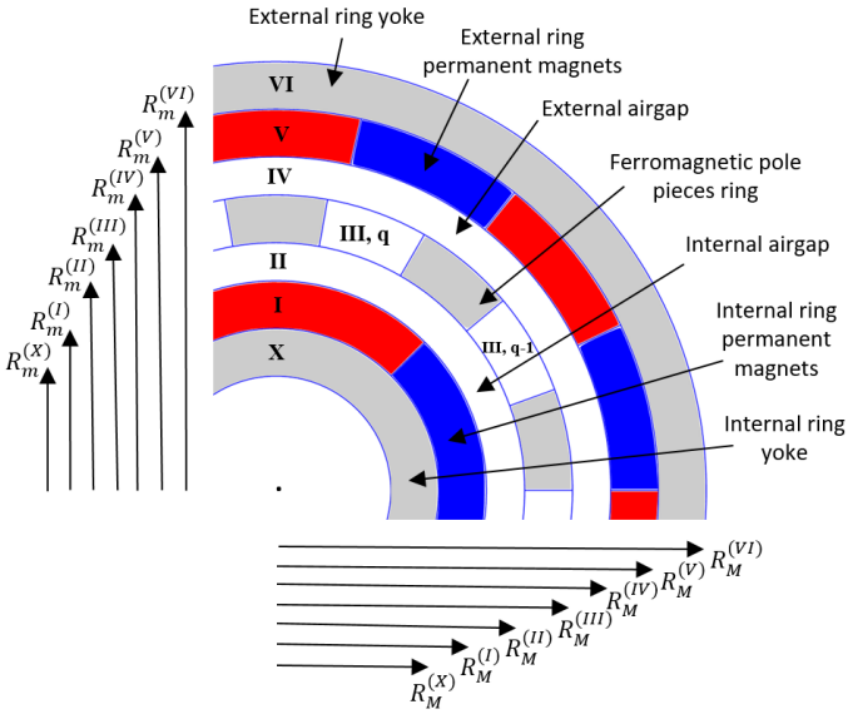

Fig. 2: Magnetic gear parameterization

$$
C_{f}=\frac{2 \cdot p_{\text {int }} \cdot Q}{N_{c}\left(2 \cdot p_{\text {int }}, Q\right)}=1
$$

\section{B. Magnetic field resolution method}

To compute a 2D magnetostatic field distribution, it is necessary to solve both Poisson's equation (6) and Laplace's equation (7) in different parts of the system [13], where $k$ is the region number defined in Fig. 2, $A^{(k)}$ and $M^{(k)}$ the magnetic vector potential and radial magnetization distribution respectively, $r$ and $\alpha$ the cylindrical coordinates, and $R_{M}^{(k)}=$ $R_{m}^{(k+1)}$ (in accordance with Fig. 2).

$$
\begin{gathered}
\frac{\partial^{2} A^{(k)}}{\partial r^{2}}+\frac{1}{r} \frac{\partial A^{(k)}}{\partial r}+\frac{\partial^{2} A^{(k)}}{\partial \alpha^{2}}=\frac{1}{r} \frac{\partial M^{(k)}}{\partial \alpha} \\
\frac{\partial^{2} A^{(k)}}{\partial r^{2}}+\frac{1}{r} \frac{\partial A^{(k)}}{\partial r}+\frac{\partial^{2} A^{(k)}}{\partial \alpha^{2}}=0
\end{gathered}
$$

For the Poisson's and Laplace's equations, a general solution (8) can be found by applying the method of separation of variables, where $X_{0}^{(k)}, Y_{0}^{(k)}, C_{n}^{(k)}, D_{n}^{(k)}, K_{n}^{(k)}$ and $E_{n}^{(k)}$ are integration constants, $a_{n}^{(k)}$ and $b_{n}^{(k)}$ are the particular solution of the equation (6). This expression shows that the number of integration constants increases as a function of the number of harmonics taken into account. For region III, which corresponds to the air space between pole pieces, the analytical solution is (9), where $\beta$ is the slot opening angle and $\alpha_{q}$ is described in (10) with $1<q<Q$.

$$
\begin{aligned}
& A^{(k)}(r, \alpha)=X_{0}^{(k)}+Y_{0}^{(k)} \ln \left(\frac{r}{R_{M}^{(k)}}\right) \\
& +\sum_{n \geq 1}^{N}\left(C_{n}^{(k)}\left(\frac{r}{R_{M}^{(k)}}\right)^{n}+D_{n}^{(k)}\left(\frac{r}{R_{m}^{(k)}}\right)^{-n}+a_{n}^{(k)}\right) \cos (n . \alpha) \\
& +\sum_{n \geq 1}^{N}\left(K_{n}^{(k)}\left(\frac{r}{R_{M}^{(k)}}\right)^{n}+E_{n}^{(k)}\left(\frac{r}{R_{m}^{(k)}}\right)^{-n}+b_{n}^{(k)}\right) \sin (n . \alpha)
\end{aligned}
$$




$$
\begin{gathered}
A^{(I I I, q)}(r, \alpha)=X_{0}^{(I I I, q)}+Y_{0}^{(I I I, q)} \ln \left(\frac{r}{R_{M}^{(I I I, q)}}\right) \\
+\sum_{m \geq 1}^{N_{Q}}\left(C_{m}^{(I I I, q)}\left(\frac{r}{R_{M}^{(I I I, q)}}\right)^{m \bar{\beta}}+D_{m}^{(I I I, q)}\left(\frac{r}{R_{m}^{(I I I, q)}}\right)^{-m \bar{\beta}}\right) \\
\cdot \cos \left(m \frac{\pi}{\beta}\left(\alpha-\alpha_{q}\right)\right) \\
\alpha_{q}=-\frac{\beta}{2}+\frac{2 \cdot q \cdot \pi}{Q}
\end{gathered}
$$

For the various problem boundaries, conditions should be given in one of the equations presented in (11). From these boundary conditions, it is possible to define a matrix system $Z$ to be inverted for determining the integration constants in several subdomains. Once the magnetic potential vector has been determined, the magnetic torque can be computed with (12) and (13), where: radius $R_{m}^{(k)}<R<R_{M}^{(k)}, B_{r}^{(k)}$ is the radial flux, $B_{\alpha}^{(k)}$ the tangential flux.

$$
\begin{aligned}
& \left\{\begin{array}{c}
\left.\frac{\partial A^{(k)}}{\partial r}\right|_{r=R_{m}^{(k)}}=0 \\
\left.\frac{\partial A^{(k)}}{\partial \alpha}\right|_{\alpha=\alpha_{q}}=0 \\
\left.\frac{\partial A^{(k)}}{\partial r}\right|_{r=R_{M}^{(k)}}=\left.\frac{\partial A^{(k+1)}}{\partial r}\right|_{r=R_{m}^{(k+1)}} \\
\left.A^{(k)}\right|_{r=R_{m}^{(k)}}=\left.A^{(k+1)}\right|_{r=R_{M}^{(k+1)}}
\end{array}\right. \\
& T^{(k)}=\frac{L_{z} \cdot R^{2}}{\mu_{0}} \sum_{n \geq 1} \int_{0}^{2 \pi} B_{r}^{(k)}(R, \alpha) \cdot B_{\alpha}^{(k)}(R, \alpha) d \alpha \\
& \left\{\begin{array}{l}
B_{r}^{(k)}=\frac{1}{r} \frac{\partial A^{(k)}}{\partial \alpha} \\
B_{\alpha}^{(k)}=-\frac{\partial A^{(k)}}{\partial r}
\end{array}\right.
\end{aligned}
$$

\section{ANALYTICAL Model With AN INFINITE RELATIVE PERMEABILITY OF YOKES (MODEL 1)}

The first analytical model developed in this part of the paper is based on an infinite relative permeability of yokes; it was proposed in [10]. To determine the integration constants for the various subsystems for all harmonics, it becomes necessary to solve a matrix system $Z_{1}$ with a number of equations (which come from the boundary conditions) equal to the number of integration constants.

When the model contains yokes with infinite relative permeability, the model is simplified and the total number of integration constants is reduced. Boundary conditions (14) have been used here to remove some integration constants given in the Appendix.

$$
\left\{\begin{array}{l}
\left.\frac{\partial A^{(I)}}{\partial r}\right|_{r=R_{m}^{(I)}}=0 \\
\left.\frac{\partial A^{(V)}}{\partial r}\right|_{r=R_{M}^{(V)}}=0
\end{array}\right.
$$

With this simplification (infinite relative permeability of the yoke), the dimension of matrix system $Z_{1}$ can be proposed in (15), where $N_{Q}$ is the number of harmonics taken into account in the air space between pole pieces (region III), $Q$ the number of pole pieces, and $N$ the number of harmonics taken into account in the other regions (i.e. I, II, IV and V) [11]. If matrix system $Z_{1}$ is inverted, it becomes possible to compute the magnetic potential vector in every region of the problem.

$$
\operatorname{Dim}\left(Z_{1}\right)=\left(12 N+\left(2 N_{Q}+2\right) Q\right)^{2}
$$

\section{Analytical Model With a Finite Relative PERMEABILITY OF YOKES (MODEL 2)}

As opposed to the previous model (i.e. with an infinite relative permeability of yokes), this $2^{\text {nd }}$ analytical model includes yokes with a finite relative permeability, which could be important to compute a global optimization proposed in [9]. Taking yokes into consideration will inevitably increase the dimension of matrix $Z_{2}$ with integration constants in regions $\mathrm{X}$ and VI defined in Fig. 2. Integration constants $D_{n}^{(k)}$ and $E_{n}^{(k)}$ of regions $\mathrm{I}$ and $\mathrm{V}$ will also increase the dimension of $Z_{2}$ since they are not simplified by the relations in (14). This approach generates $8 \mathrm{~N}$ more integration constants than the previous analytical model which induces an increase of the matrix system $Z_{2}$ dimension to be inverted for determining the magnetic field distribution in previous regions of the problem and yoke regions (see Equation (16)).

$$
\operatorname{Dim}\left(Z_{2}\right)=\left(20 N+\left(2 N_{Q}+2\right) Q\right)^{2}
$$

To obtain the magnetic field distribution, new boundary conditions must be taken into account for regions $\mathrm{X}, \mathrm{I}, \mathrm{V}$ and VI. The boundary conditions between regions $\mathrm{X}$ and $\mathrm{I}$ and between regions V and VI (14) are replaced by (17) (the relative permeability of yokes is no longer infinite, but equal to 1000 , while the relative permeability of pole pieces remains infinite). On the inner radius of region $\mathrm{X}$ and on the outer radius of region VI, boundary conditions (18) are imposed.

$$
\left\{\begin{aligned}
\left.\frac{\partial A^{(X)}}{\partial \alpha}\right|_{r=R_{m}^{(I)}} & =\left.\frac{\partial A^{(I)}}{\partial \alpha}\right|_{r=R_{m}^{(I)}} \\
\left.\frac{1}{\mu_{X}} \cdot \frac{\partial A^{(X)}}{\partial r}\right|_{r=R_{m}^{(I)}} & =\left.\frac{1}{\mu_{I}} \cdot \frac{\partial A^{(I)}}{\partial r}\right|_{r=R_{m}^{(I)}} \\
\left.\frac{\partial A^{(V)}}{\partial \alpha}\right|_{r=R_{M}^{(V)}} & =\left.\frac{\partial A^{(V I)}}{\partial \alpha}\right|_{r=R_{M}^{(V)}} \\
\left.\frac{1}{\mu_{V}} \cdot \frac{\partial A^{(V)}}{\partial r}\right|_{r=R_{M}^{(V)}} & =\left.\frac{1}{\mu_{V I}} \cdot \frac{\partial A^{(V I)}}{\partial r}\right|_{r=R_{M}^{(V)}}
\end{aligned}\right.
$$




$$
\left\{\begin{array}{l}
\left.A^{(X)}\right|_{r=R_{m}^{(X)}}=0 \\
\left.A^{(V I)}\right|_{r=R_{M}^{(V I)}}=0
\end{array}\right.
$$

This consideration leads to the $8 N$ equations given in the Appendix, which are required to transition from analytical model 1 to analytical model 2 .

Like the other model, when matrix system $Z_{2}$ is inverted, it becomes possible to draw the magnetic flux line distribution in previous regions of the problem and in yokes regions as shown in Fig. 3.

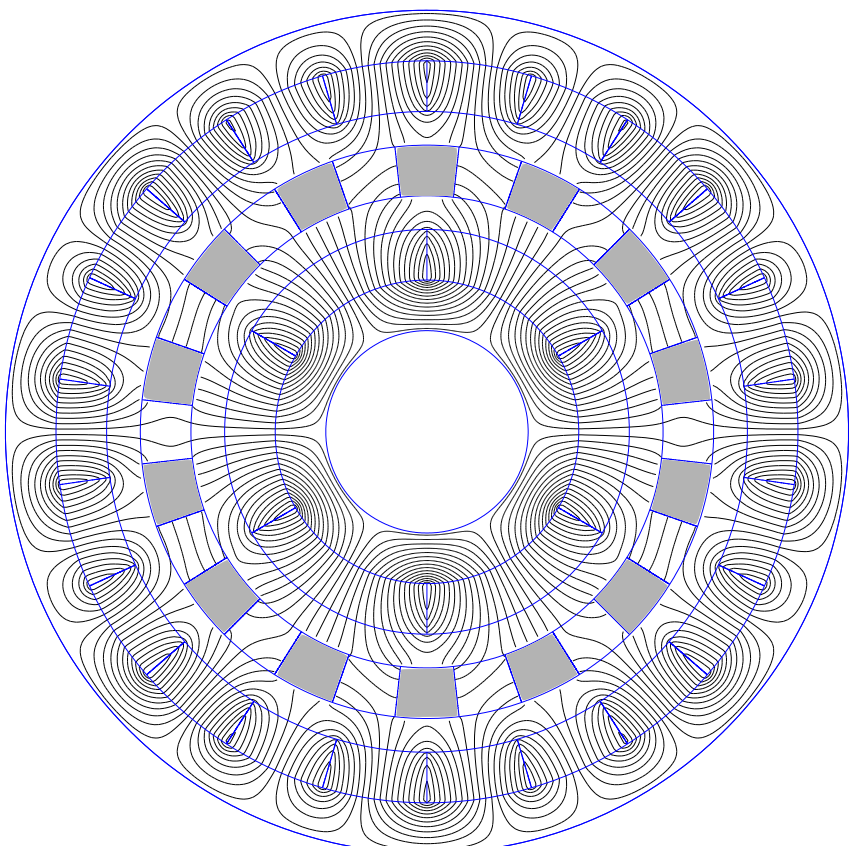

Fig. 3: Magnetic flux line distribution obtained with analytical model 2 (with finite relative permeability of yokes)

\section{HARMONIC SELECTION METHODS}

In this section and the following one, the studies have been based on an example with a high pole number, as described in Table I, which is similar to [9] in respecting condition (5) (cogging torque factor equal to 1). For both previous models and harmonic selection methods, the matrix system dimension $Z_{i, j}$ will be compared with $N_{Q}=N / p_{\text {ext }}=5$, where $i$ assumes the values 1,2 and $j$ assumes the values $A, B, C, D$. To compare the efficiency of the various harmonic selection methods, the product $B_{r} . B_{\alpha}$ spectrum, which generates the magnetic torque obtained in (11), will be analyzed in Fig. 4.

\section{Method A: Initial method without harmonic selection ("Exact" analytical method)}

The product $B_{r}$. $B_{\alpha}$ spectrum obtained using the analytical model without a harmonic selection method is presented in Fig. 4 with $N_{Q}=N / p_{\text {ext }}=10$. The initial method [10] allows taking into account all harmonic systems, from the lowest periodicity $2 \pi$ to the highest, i.e. 5. $p_{\text {ext }} .2 \pi$ in regions X, I, II, IV, V and VI (specified in Fig. 2). Let's observe that many system harmonics do not generate magnetic torque and can be removed from the magnetic field computation. This method
TABLE I

DATA FOR THE MAGNETIC GEAR EXAMPLE IN SECTION VI

\begin{tabular}{|c|c|c|}
\hline Symbol & Quantity & Value \\
\hline$p_{\text {int }}$ & Number of internal ring pole pairs & 20 \\
\hline$p_{\text {ext }}$ & Number of external ring pole pairs & 131 \\
\hline$Q$ & Number of ferromagnetic pole pieces & 151 \\
\hline$C_{f}$ & Cogging torque factor & 1 \\
\hline$D$ & External diameter & $4 \mathrm{~m}$ \\
\hline$\delta_{\text {int }}$ & Internal air gap & $5 \mathrm{~mm}$ \\
\hline$\delta_{\text {ext }}$ & External air gap & $5 \mathrm{~mm}$ \\
\hline$e_{\text {yoke int }}$ & Radial thickness of the internal ring yoke & $106 \mathrm{~mm}$ \\
\hline$e_{\text {yoke ext }}$ & Radial thickness of the external ring yoke & $65 \mathrm{~mm}$ \\
\hline$e_{P M \text { int }}$ & $\begin{array}{l}\text { Radial thickness of the internal ring permanent } \\
\text { magnets }\end{array}$ & $19 \mathrm{~mm}$ \\
\hline$e_{P M ~ e x t}$ & $\begin{array}{l}\text { Radial thickness of the external ring permanent } \\
\text { magnets }\end{array}$ & $10 \mathrm{~mm}$ \\
\hline$e_{s}$ & Radial thickness of ferromagnetic pole pieces & $52 \mathrm{~mm}$ \\
\hline$L_{z}$ & Magnetic length of the magnetic gear & $2.1 \mathrm{~m}$ \\
\hline$B_{r}$ & Remanence of magnets & $1.2 \mathrm{~T}$ \\
\hline$\mu_{I}-\mu_{V}$ & Relative permeability of magnets & 1 \\
\hline$\mu_{X}-\mu_{V I}$ & Relative permeability of yokes (with Model 1) & $\infty$ \\
\hline$\mu_{X}-\mu_{V I}$ & Relative permeability of yokes (with Model 2) & 1,000 \\
\hline
\end{tabular}

incorporates 5 external ring harmonics and 5. $\left\lfloor p_{\text {ext }} / p_{\text {int }}\right\rfloor$ internal ring harmonics. To reduce the dimension of matrix system $Z_{i, j}$ while maintaining the same level of accuracy, harmonic systems may be taken into account from periodicity $p_{\text {int }} .2 \pi$ to $5 . p_{\text {ext }} \cdot 2 \pi$ [11]. The dimension of matrix system $Z_{1, A}$ and $Z_{2, A}$ is then given by (19) and (20) respectively for the magnetic gear described in Table I. Without any harmonic selection method, the matrix dimension is increased substantially for Model 2 (with a finite relative permeability of the yokes).

$$
\begin{gathered}
\operatorname{Dim}\left(Z_{1, A}\right)=9444^{2} \\
\operatorname{Dim}\left(Z_{2, A}\right)=14532^{2}
\end{gathered}
$$

Method B: Ring harmonics and particular modulated harmonics selection

As shown in the Fig. 4 for the "exact" method, some of the presented harmonics do not correspond to either the internal or external ring harmonics, but they do correspond to other harmonics due to modulation. To take into account the main harmonics of the system, this harmonics selection method takes into account (for regions other than III):

- the impaired harmonics of the permanent magnet rings

- fundamental components of the internal and external rings modulated by pole piece ring harmonics (e.g. harmonics with a periodicity of $\left.\left(3 . Q-p_{\text {int }}\right) \cdot 2 \pi\right)$

- the first impaired harmonic of internal and external rings modulated by poles pieces ring fundamental (e.g. harmonics with a periodicity of $\left.\left(Q-3 . p_{i n t}\right) \cdot 2 \pi\right)$.

According to this method, the dimension of matrix systems $Z_{1, B}$ and $Z_{2, B}$ is considerably reduced, as revealed in Equations (21) and (22). With this harmonic selection method, the dimensions of matrix $Z_{2, B}$ do not increase substantially compared to matrix $Z_{1, B}$, as opposed to method A (equations 
(19)-(20)). This method serves to reduce the matrix dimension, hence computation time, without any loss of accuracy because every significant harmonic has been selected, as shown in Fig. 4 (only 12 harmonics are taken into account vs. approximately 500 with method A).

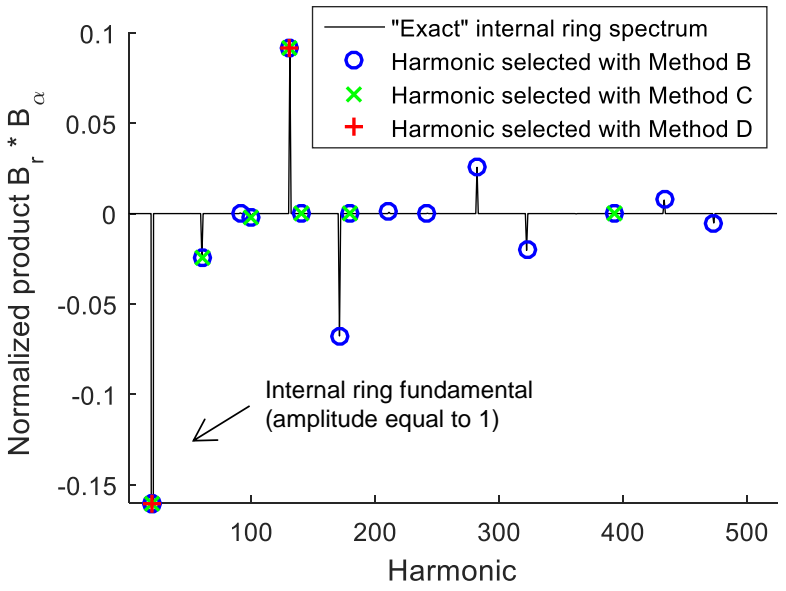

(a)

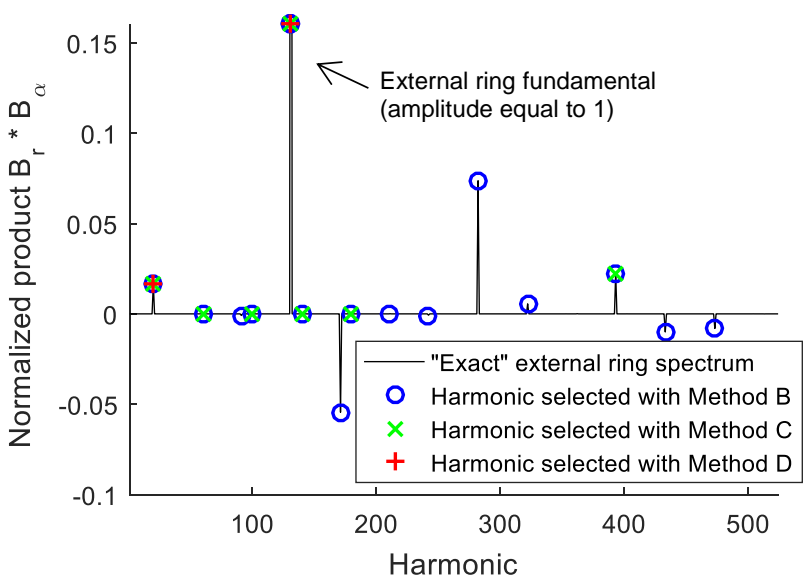

(b)

Fig. 4: Illustration of the various harmonic selection methods with the normalized $B_{r} . B_{\alpha}$ product spectrum obtained using the exact analytical method (with the finite relative permeability of yokes) for the magnetic gear described in Table I, at: (a) the middle of the external air gap, and (b) the middle of the internal air gap

$$
\begin{aligned}
& \operatorname{Dim}\left(Z_{1, B}\right)=2100^{2} \\
& \operatorname{Dim}\left(Z_{2, B}\right)=2292^{2}
\end{aligned}
$$

Method C: Only permanent magnets impairing harmonics selection

To further reduce the matrix dimension, this harmonic selection method only targeted the impaired harmonics of the permanent magnet rings. According to this method, the dimension of the matrix system $Z_{1, C}$ and $Z_{2, C}$ is given by (23) and (24) respectively. This method further reduces computation time, although precision may be lowered as well because every significant harmonic has not been selected, as shown in Figure 4.

$$
\begin{aligned}
& \operatorname{Dim}\left(Z_{1, C}\right)=1908^{2} \\
& \operatorname{Dim}\left(Z_{2, C}\right)=1972^{2}
\end{aligned}
$$

\section{Method D: Fundamental selection of just permanent magnets}

To further reduce the matrix dimension beyond that of method $\mathrm{C}$, this last harmonic selection method only targets the fundamentals of the permanent magnet rings. According to this method, the dimension of matrix system $Z_{1, C}$ and $Z_{2, C}$ is given in (25) and (26), respectively. With this method D, the level of precision may be further lowered compared to method $\mathrm{C}$ (see Fig. 4).

$$
\begin{aligned}
& \operatorname{Dim}\left(Z_{1, D}\right)=1836^{2} \\
& \operatorname{Dim}\left(Z_{2, D}\right)=1852^{2}
\end{aligned}
$$

\section{COMPUTATION TIME AND PRECISION COMPARISON}

\section{A. Torque ripple comparison}

During an optimization phase needed for system development, some system properties must be evaluated quickly (on the order of a second) with relative precision for the various system configurations [14]. In the context of magnetic gear optimization, the main property to evaluate is the maximum gear torque [15], which is obtained for a load angle equal to $\pi / 2$ with an amount of torque ripple that depends on the pole configuration [5]. Fig. 5 indicates that with respect to (5) (cogging torque factor $C_{f}=1$ ), the magnetic torque can only be evaluated for a single position corresponding to the maximum gear torque (with a load angle equal to $\pi / 2$ ). This criterion is then used to compare computation times for the various models and various harmonic selection methods in the next section.

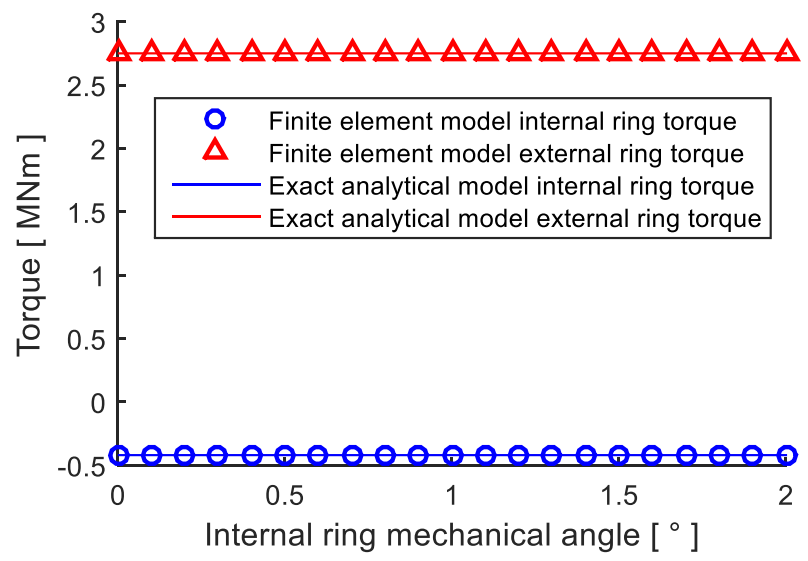

Fig. 5: Magnetic torque evolution obtained using Method A with finite relative permeability of yokes and a finite element model for the magnetic gear described in Table I. A $2^{\circ}$ rotation for the internal ring and $13.1^{\circ}$ for the external ring (configuration where $\omega_{Q / 0}=0$ ) has been considered.

B. Analysis of the number of harmonics taken into account in pole piece regions $\left(N_{Q}\right)$

To reduce computation time, different approximations were performed, which on all occasions were detrimental to 
precision. A compromise between computation time and precision must be found before optimization for the range of approximations presented above (in knowing that the yokes consideration from these analytical models do impact computation time but not precision).

To evaluate the level of precision, the torque value reference $T_{\text {ref }}^{(I V)}$ has been computed using model 2 without any harmonic selection (i.e. Method A), with $N_{Q}=N / p_{\text {ext }}=10$ and considering the torque of the external permanent magnet ring. A preliminary analysis of the impact of $N_{Q}$, i.e. the number of harmonics taken into account in region III, has been conducted for various values of $N$, i.e. the maximum value of system harmonics in the other regions. It is then possible to analyze the computation time and precision of the torque evaluation without having to proceed with harmonic selection (Method A), as shown in Fig. 6 with the error defined in (27), for the harmonic selection method $i=A$.

$$
\text { Error }=\left|\frac{T_{\text {ref }}^{(I V)}-T^{(I V)}\left(N_{Q}, \frac{N}{p_{e x t}}, i\right)}{T_{\text {ref }}^{(I V)}}\right|
$$
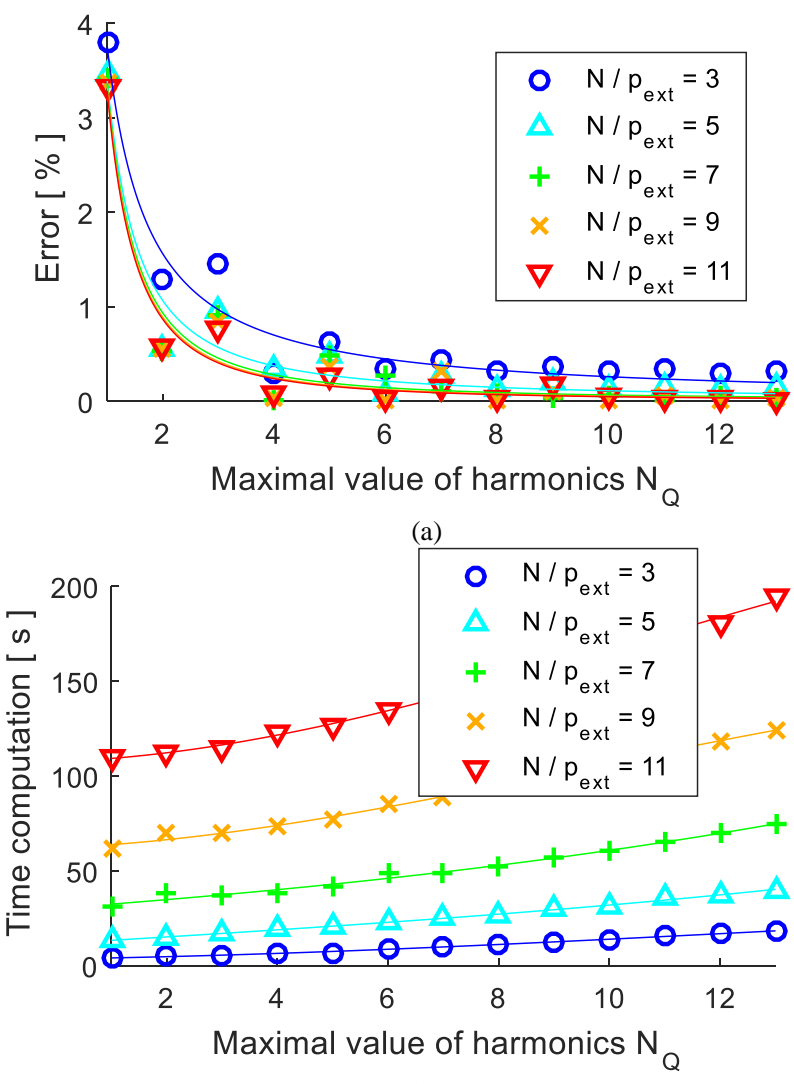

(b)

Fig. 6: Torque evaluation for various values of $N$ vs. $N_{Q}$ without harmonic selection (Method A) for the magnetic gear described in Table I: (a) precision, and (b) computation time

Fig. 6 indicates that the precision and computation time increase as a function of $N_{Q}$ and $N$. However, regardless of the value of $N$, it was observed that the torque evaluation error remained less than $1 \%$ with a safety margin with $N_{Q}=5$. It will thus be assumed for the rest of this study that the maximum value of pole piece harmonics in region III $N_{Q}$ is fixed and equal to 5 . This consideration is not yet sufficient to reduce the computation time for optimization. It then becomes necessary to analyze the impact of the harmonic selection methods on both computation time and precision.

\section{Computation time and precision analysis for various harmonic selection methods}

To reduce computation time, the dimension of matrix system $Z_{i, j}$ may be reduced with harmonic selection methods. It is then possible to analyze the computation time and precision of the torque evaluation for the various harmonic selection methods in function of $N$, the maximal value of system harmonics with $N_{Q}=5$, as shown in Fig. 7 (the torque value reference is still $T_{r e f}^{(I V)}$, as presented before).

Fig. 7 illustrates that harmonic selection methods B, C and D reduce computation time considerably with an error (defined in (27), $N_{Q}=5$ ) of below $1 \%$ (for $N / p_{\text {ext }} \neq 1$ ) for method B, less than $4 \%$ for method $\mathrm{C}$ and less than $5 \%$ for method $\mathrm{D}$. The best compromise found between computation time and precision is thus to apply harmonic selection method B with $N / p_{\text {ext }}=3$ and $N_{Q}=5$, which permits to have a torque evaluation in 0.36 seconds (with an Intel Xeon E5-1630 v3, 8 threads, $3.70 \mathrm{GHz}$ ) and an error of less than $1 \%$ using the analytical model with a finite relative permeability of yokes.

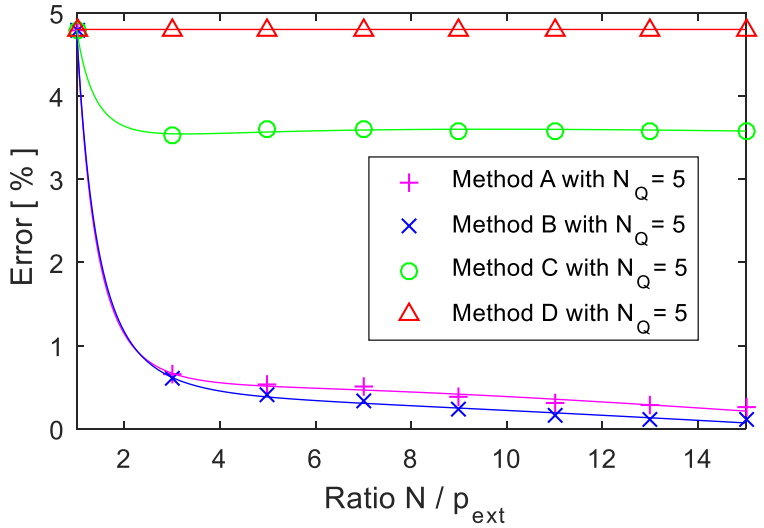

(a)

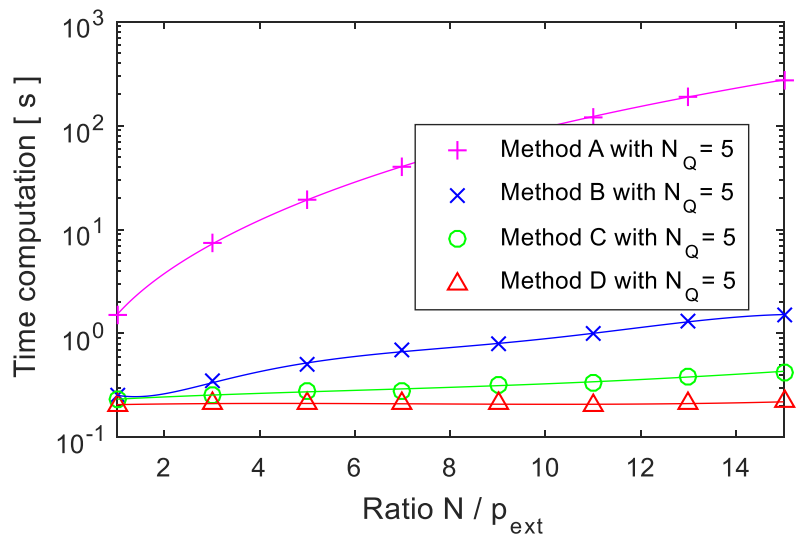

(b)

Fig. 7: Torque evaluation for various harmonic selection methods vs. harmonic number $N$, computed with an ideal yoke and $N_{Q}=5$ for the magnetic gear described in Table I: (a) precision, and (b) computation time 
D. Influence of the yokes consideration (finite or infinite relative permeability) in the analytical models

Finite relative permeability of yokes consideration will increase the matrix system $Z_{i, j}$ dimension and hence computation time. However, for a global system optimization, it may be necessary to include yokes in the procedure. It is then possible to analyze the computation time of the torque evaluation obtained using the analytical model with a finite relative permeability of yokes (model 2) and compare it with different models. Table II presents this comparison for the various models (the level of precision has not been presented since yokes consideration has almost no impact on precision).

TABLE II

COMPUTATION TIME COMPARISON

\begin{tabular}{|c|c|c|c|c|c|c|}
\hline \multirow{2}{*}{} & \multicolumn{2}{|c|}{$\begin{array}{c}\text { Harmonic } \\
\text { selection } \\
\text { method A }\end{array}$} & \multicolumn{2}{c|}{$\begin{array}{c}\text { Harmonic } \\
\text { selection } \\
\text { method B }\end{array}$} & \multicolumn{2}{|c|}{$\begin{array}{c}\text { Finite element } \\
\text { model }\end{array}$} \\
\cline { 2 - 7 } & $\begin{array}{c}\text { Model } \\
2 \\
(\mathrm{sec})\end{array}$ & $\begin{array}{c}\text { Model } \\
(\mathrm{sec})\end{array}$ & $\begin{array}{c}\text { Model } \\
2\end{array}$ & $\begin{array}{c}\text { Model } \\
(\mathrm{sec})\end{array}$ & $\begin{array}{c}4 \text { elt. per } \\
\text { airgap } \\
(\mathrm{sec})\end{array}$ & $\begin{array}{c}1 \text { elt. per } \\
\text { airgap } \\
(\mathrm{sec})\end{array}$ \\
\hline $\begin{array}{c}N / p_{\text {ext }}=10 \\
N_{Q}=10\end{array}$ & 480 & 145 & 2.6 & 2.0 & 320 & 180 \\
\hline $\begin{array}{c}N / p_{\text {ext }}=3 \\
N_{Q}=5\end{array}$ & 22.3 & 7.5 & 0.36 & 0.32 & 320 & 180 \\
\hline
\end{tabular}

Table II indicates that for a high pole number magnetic gear, the analytical model computation time can exceed the finite element computation time without any harmonic selection and with $N_{Q}=N / p_{\text {ext }}=10(180 \mathrm{sec}$ vs. $480 \mathrm{sec})$. This finite element analysis included 1.1 million elements and four elements per air gap for the first column, and 0.6 million elements and one element per air gap for the second column; it was conducted with the FEMM 4.2 software. In contrast, it has been observed that integrating finite relative permeability of yoke into the analytical model slightly increases computation time with harmonic selection method B $(0.36 \mathrm{sec}$ vs. $0.32 \mathrm{sec}$ with $N / p_{\text {ext }}=3$ and $N_{Q}=5$ ) for the same level of precision. The best compromise between computation time and precision has been obtained using method $B$ with $N / p_{\text {ext }}=3$ and $N_{Q}=$ 5 and the robustness of this solution can be evaluated.

\section{ROBUSTNESS OF HARMONIC SELECTION METHOd B}

A. Torque computation for other magnetic gear configurations

In order to validate the harmonic selection method B for various magnetic gear geometries, an analysis of the computation time and error (defined in equation (27)) is conducted for the different pole numbers presented in Table III, with a finite relative permeability of the yoke (with the same radial thicknesses as in the magnetic gear studied above): $N / p_{\text {ext }}=3$ and $N_{Q}=5$.
TABLE III

COMPUTATION TIME AND PRECISION ANALYSIS FOR VARIOUS MAGNETIC Gear TOPOLOGIES WITH HARMONIC SELECTION METHOD B AND

\begin{tabular}{|c|c|c|c|c|}
\multicolumn{5}{c}{$N / p_{\text {ext }}=3$ AND $N_{Q}=5$} \\
\hline$p_{\text {int }}$ & Config. 1 & Config. 2 & Config. 3 & Config. 4 \\
\hline$p_{\text {ext }}$ & 134 & 20 & 20 & 20 \\
\hline$Q$ & 154 & 155 & 130 & 120 \\
\hline$C_{f}$ & 2 & 5 & 10 & 140 \\
\hline $\begin{array}{c}\text { Computation } \\
\text { time (sec) }\end{array}$ & 0.33 & 0.33 & 0.32 & 0.27 \\
\hline Error (\%) & 0.7 & 0.4 & 0.6 & 0.8 \\
\hline
\end{tabular}

For these various magnetic gear topologies, the error on the torque computation lies between $0.4 \%$ and $0.8 \%$, with a computation time of between $0.27 \mathrm{sec}$ and $0.33 \mathrm{sec}$. Table III shows that the precision and computation time do not differ much for the studied configurations. This finding validates the general use of harmonic selection method B. However, for a global optimization, the torque ripple is a problem if just one position is evaluated.

\section{B. Evaluation of other magnetic gear properties}

The harmonic selection method proposed in this article (Method B) enables significantly reducing the computation time of the magnetic torque without any loss of accuracy. It can also be used to compute other properties of the magnetic gear, like eddy current losses in permanent magnets [16], iron losses in yokes [17] and tangential and radial loads on pole pieces. The computation of these properties is solely based on the computation of radial and tangential components of the flux density distribution for the different regions.

In order to evaluate the relevance of harmonic selection method B for the calculation of the aforementioned properties, it is simply necessary to control the flux density distribution computation in the different regions. Fig. 8 shows the flux density distribution in the magnetic gear obtained with harmonic selection method B, with $N_{Q}=N / p_{\text {ext }}=10$ for the magnetic gear presented in Table I. A comparison can be drawn with the "exact" analytical model (Method A), and the flux density distribution difference between the two methods can be computed for every point of the different regions, as shown in Figure 9. Table IV lists the average differences of the flux density distribution obtained in these regions with $N_{Q}=$ $N / p_{\text {ext }}=10$. 


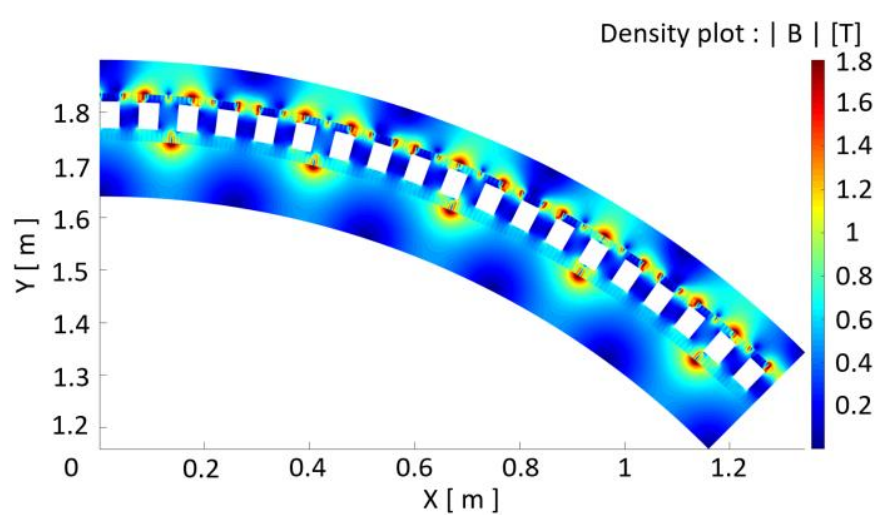

Fig. 8: Flux density distribution obtained with harmonic selection method $\mathrm{B}$ and $N_{Q}=N / p_{\text {ext }}=10$ for the magnetic gear presented Table I.

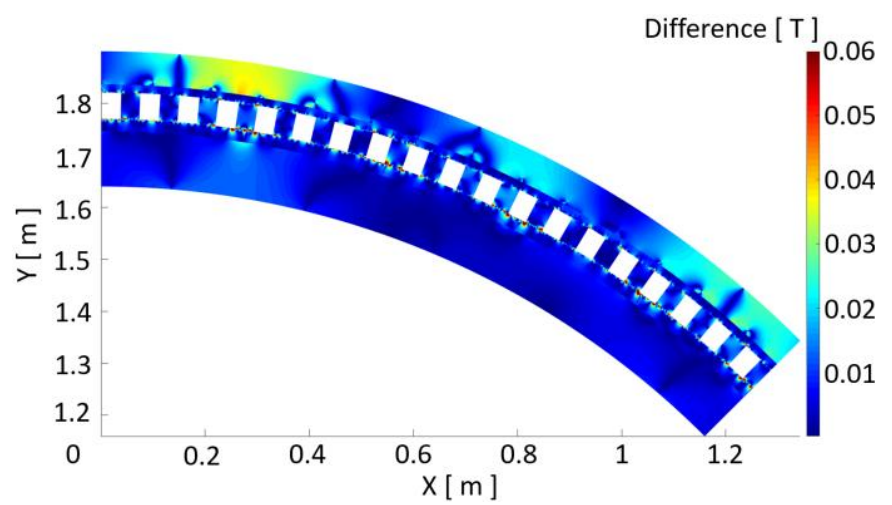

Fig. 9: Flux density distribution difference between harmonic selection method B and the exact analytical model with $N_{Q}=N / p_{\text {ext }}=10$ for the magnetic gear presented Table I

TABLE IV

AVERAGE DiFFERENCE BETWEEN THE TWO FLUX DENSITY DistRIBUTION METHODS OBTAINED IN DIFFERENT REGIONS WITH $N_{Q}=N / p_{\text {ext }}=10$

\begin{tabular}{|c|c|c|c|c|c|c|c|}
\hline & $\begin{array}{c}\text { Reg. } \\
\text { X }\end{array}$ & $\begin{array}{c}\text { Reg. } \\
\text { I }\end{array}$ & $\begin{array}{c}\text { Reg. } \\
\text { II }\end{array}$ & $\begin{array}{c}\text { Reg. } \\
\text { III }\end{array}$ & $\begin{array}{c}\text { Reg. } \\
\text { IV }\end{array}$ & $\begin{array}{c}\text { Reg. } \\
\text { V }\end{array}$ & $\begin{array}{c}\text { Reg. } \\
\text { VI }\end{array}$ \\
\hline Av. & & & & & & & \\
diff. & 5 & 6 & 26 & 9 & 10 & 7 & 2 \\
x10 & & & & & & & \\
\hline
\end{tabular}

Table IV shows that harmonic selection method B enables computing the flux density distribution with a total average difference between method $\mathrm{A}$ and $\mathrm{B}$ of $0.012 \mathrm{~T}$ across the different regions of the magnetic gear with $N_{Q}=N / p_{\text {ext }}=$ 10. This finding validates the use of harmonic selection method $\mathrm{B}$ to compute the different magnetic gear properties (eddy current losses in permanent magnets, iron losses in yokes, and the tangential and radial loads on pole pieces) in the magnetic gears.

In order to minimize computation time and maintain an acceptable precision for the other magnetic gear properties, $N_{Q}$, the number of harmonics taken into account in the air space between pole pieces (region III), and $N$, the number of harmonics taken into account in the other regions (i.e. I, II, IV and V), can both be modified. It then becomes necessary to analyze the error on the final value of the evaluated property (losses and magneto-mechanical loads) and not solely on the flux density distribution values. It is indeed possible to generate a sizable error on the flux density distribution with an acceptable error on the final value of the evaluated properties. This condition implies that the values of $N_{Q}$ and $N$, which correspond to the best compromise between computation time and precision, should be determined for the various magnetic gear property evaluations (losses and magneto-mechanical loads).

\section{CONCLUSION}

This paper has described a novel model for analyzing magnetic field distribution that includes a finite relative permeability of yokes (model 2) for both the internal and external permanent magnet rings of a magnetic gear. For this model and the other one with an infinite relative permeability of yokes (model 1) [10], harmonic selection methods have been proposed and compared in order to reduce the computation time required to determine the magnetic torque for one magnetic configuration of a high pole number magnetic gear.

The result of this comparison suggests the possibility of significantly reducing computation time with harmonic selection method $B$, which selects impaired harmonics of the permanent magnet rings along with particular modulated harmonics of the system (has describe section VI) for regions $\mathrm{X}, \mathrm{I}, \mathrm{II}, \mathrm{IV}, \mathrm{V}$ and VI, as defined in Fig. 2. The best compromise between computation time and precision has been obtained using method $B$ with $N / p_{\text {ext }}=3$ and $N_{Q}=5$. With these parameters, the torque evaluation is computed at a precision above $99 \%$ in $0.32 \mathrm{sec}$ (for the magnetic gear presented in Table I with an Intel Xeon E5-1630 v3, 8 threads, $3.70 \mathrm{GHz}$ ) for model 1 and in $0.36 \mathrm{sec}$ for model 2 . Analytical model 2 and harmonic selection method $B$ divide the computation time by 900 relative to a 2D finite element model and by 60 compared to the exact analytical method (Method $A$ with $N / p_{\text {ext }}=3$ and $N_{Q}=5$ and analytical model 2) with an error below $1 \%$. With this model, it would be possible to achieve a strong optimization of a high pole number magnetic gear with an acceptable computation time (less than one day).

Looking forward, the harmonic selection method proposed in this article will be used to more quickly compute the various mechanical loads in the magnetic gear and the eddy current losses in permanent magnets (Table IV). The analytical model with a finite relative permeability of yokes will also be able to compute iron losses in yokes (in laminated magnetic material). An analysis of the number of harmonics taken into account in the various regions ( $N$ and $N_{Q}$ ) remains to be completed in order to minimize computation time for the magnetic gear properties other than torque.

\section{APPENDIX}

Equations (28) and (29), which serve to remove some integration constants from (14) in region I with (28) and in region $\mathrm{V}$ with (29) ((14) generates a condition between $C_{n}^{(k)}$ and $D_{n}^{(k)}$, and between $K_{n}^{(k)}$ and $\left.E_{n}^{(k)}\right)$. 


$$
\begin{aligned}
& \left\{\begin{array}{l}
D_{n}^{(I)}=\left(\frac{R_{m}^{(I)}}{R_{M}^{(I)}}\right)^{n} C_{n}^{(I)} \\
E_{n}^{(I)}=\left(\frac{R_{m}^{(I)}}{R_{M}^{(I)}}\right)^{n} K_{n}^{(I)}
\end{array}\right. \\
& \left\{\begin{array}{l}
D_{n}^{(V)}=\left(\frac{R_{M}^{(V)}}{R_{m}^{(V)}}\right)^{n} C_{n}^{(V)} \\
E_{n}^{(V)}=\left(\frac{R_{M}^{(V)}}{R_{m}^{V}}\right)^{n} K_{n}^{(V)}
\end{array}\right.
\end{aligned}
$$

The $8 N$ equations (30)-(37) identified to transition from an analytical model with infinite relative permeability of yokes to the analytical model with finite relative permeability of yokes which come from (17)-(18), i.e.:

$$
\begin{gathered}
C_{n}^{(X)}\left(1-\left(\frac{R_{m}^{(X)}}{R_{M}^{(X)}}\right)^{2 n}\right)-C_{n}^{(I)}\left(\frac{R_{m}^{(I)}}{R_{M}^{(I)}}\right)^{n}-D_{n}^{(I)}=a_{n}^{(I)} \\
K_{n}^{(X)}\left(1-\left(\frac{R_{m}^{(X)}}{R_{M}^{(X)}}\right)^{2 n}\right)-K_{n}^{(I)}\left(\frac{R_{m}^{(I)}}{R_{M}^{(I)}}\right)^{n}-E_{n}^{(I)}=b_{n}^{(I)} \\
C_{n}^{(X)}\left(1+\left(\frac{R_{m}^{(X)}}{R_{M}^{(X)}}\right)^{2 n}\right) \frac{\mu_{I}}{\mu_{X}}-C_{n}^{(I)}\left(\frac{R_{m}^{(I)}}{R_{M}^{(I)}}\right)^{n}+D_{n}^{(I)}=0 \\
K_{n}^{(X)}\left(1+\left(\frac{R_{m}^{(X)}}{R_{M}^{(X)}}\right)^{2 n}\right) \frac{\mu_{I}}{\mu_{X}}-K_{n}^{(I)}\left(\frac{R_{m}^{(I)}}{R_{M}^{(I)}}\right)^{n}+E_{n}^{(I)}=0 \\
D_{n}^{(V I)}\left(1-\left(\frac{R_{m}^{(V I)}}{R_{M}^{(V I)}}\right)^{-2 n}\right)-D_{n}^{(V)}\left(\frac{R_{M}^{(V)}}{R_{m}^{(V)}}\right)^{-n}-C_{n}^{(V)}=a_{n}^{(V)} \\
E_{n}^{(V I)}\left(1-\left(\frac{R_{m}^{(V I)}}{R_{M}^{(V I)}}\right)^{-2 n}\right)-E_{n}^{(V)}\left(\frac{R_{M}^{(V)}}{R_{m}^{(V)}}\right)^{-n}-K_{n}^{(V)}=b_{n}^{(V)} \\
D_{n}^{(V I)}\left(1+\left(\frac{R_{m}^{(V I)}}{R_{M}^{(V I)}}\right)^{-2 n}\right) \frac{\mu_{V}}{\mu_{V I}}-D_{n}^{(V)}\left(\frac{R_{M}^{(V)}}{R_{m}^{(V)}}\right)^{-n}+C_{n}^{(V)}=0 \\
E_{n}^{(V I)}\left(1+\left(\frac{R_{m}^{(V I)}}{R_{M}^{(V I)}}\right)^{-2 n}\right) \frac{\mu_{V}}{\mu_{V I}}-E_{n}^{(V)}\left(\frac{R_{M}^{(V)}}{R_{m}^{(V)}}\right)^{-n}+K_{n}^{(V)}=0
\end{gathered}
$$

\section{ACKNOWLEDGMENTS}

The authors would like to thank Jeumont Electric for the different discussions around the system design, with special gratitude to D. Laloy, D. Ekeom and A. Fasquelle.

\section{REFERENCES}

J. Keller, Y. Guo, and L. Sethuraman, "Gearbox Reliability Collaborative Investigation of Gearbox Motion and High-SpeedShaft Loads," NREL, Tech. Rep. TP-5000-65321, 2016.

[2] W. Teng, X. Ding, X. Zhang, Y. Liu, and Z. Ma, "Multi-fault detection and failure analysis of wind turbine gearbox using complex wavelet transform," Renew. Energy, vol. 93, pp. 591-598, 2016.
[3] K. Atallah and D. Howe, "A novel high-performance magnetic gear," IEEE Trans. Magn., vol. 37, no. 4, pp. 2844-2846, 2001.

[4] T. B. Martin, "Magnetic transmission," Pat. US3378710, 1968.

[5] K. Atallah, S. D. Calverley, and D. Howe, "Design, analysis and realisation of a high-performance magnetic gear," IEE Proc - Electr. Power Appl., vol. 151, no. 2, pp. 135-143, 2004.

[9] M. Desvaux, B. Multon, H. Ben Ahmed, R. Le Goff Latimier, and S. Sire, "Design and Optimization of Magnetic Gears with Arrangement and Mechanical Constraints for Wind Turbine Applications," in Conf. EVER'16, 2016.

[10] T. Lubin, S. Mezani, and A. Rezzoug, "Analytical computation of the magnetic field distribution in a magnetic gear," IEEE Trans. Magn., vol. 46, no. 7, pp. 2611-2621, 2010.

[11] A. Penzkofer and K. Atallah, "Magnetic Gears for High Torque Applications," IEEE Trans. Magn., vol. 50, no. 11, 2014.

[12] R. Mathis and Y. Rémond, "A unified theory of epicyclic gear trains," C. R. Acad. Sci. Paris, pp. 1115-1121, 1999.

T. Lubin, S. Mezani, and A. Rezzoug, "Exact analytical method for magnetic field computation in the air-gap of cylindrical electrical machines considering slotting effects," IEEE Trans. Magn., vol. 46, no. 4, pp. 1092-1099, 2009.

J. Aubry, H. Ben Ahmed, and B. Multon, "Sizing optimization methodology of a surface permanent magnet machine-converter system over a torque-speed operating profile: Application to a wave energy converter," IEEE Trans. Ind. Electron., vol. 59, no. 5, pp. 2116-2125, 2012.

5] S. Niu, N. Chen, S. L. Ho, and W. N. Fu, "Design optimization of magnetic gears using mesh adjustable finite-element algorithm for improved torque," IEEE Trans. Magn., vol. 48, no. 11, pp. 41564159, 2012.

O. de la Barrière, S. Hlioui, H. Ben Ahmed, M. Gabsi“'An analytical model for the computation of no-load eddy current losses in the rotor of a permanent magnet synchronous machine," vol. 52, no. 6, 2013.

C. A. Hernandez-Aramburo, T. C. Green, and A. C. Smith, "Estimating Rotational Iron Losses in an Induction Machine," IEEE Trans. Magn., vol. 39, no. 6, pp. 3527-3533, 2003.

Melaine Desvaux received a joint B.S. degree in electronics and telecommunications and in mechanical engineering in 2012, followed by an M.S. degree in mechatronic design in 2015, from the University of Rennes 1 (France). He passed the "Agrégation" credentialing exam in Mechanical Engineering in 2014 and he is currently pursuing a Ph.D. degree in mechatronic engineering at the SATIE Laboratory, ENS Rennes, France.

His research topics include the integration of magnetic gears in wind power applications, a field that requires skills in mechanical engineering, optimization and magnetism.

Benjamin Traullé received a joint B.S. degree in electronics and telecommunications and in mechanical engineering in 2015, from the University of Rennes 1 (France). He conducted a summer internship at the SATIE Laboratory, ENS Rennes (France).

Roman Le Goff Latimier received his "Agrégation" instructor's credential in Electrical Engineering in 2012. He received an M.S. degree in renewable energy in 2013, from the Ecole Polytechnique (France). He was awarded a Ph.D. degree in electrical engineering from the SATIE Laboratory, ENS Rennes (France) in 2016 for his work on the optimal sizing and management of renewable sources and electric vehicle fleets.

His research interests include stochastic optimization energy forecasting for smart grids with a large penetration of stochastic and distributed agents. 
Stéphane Sire received his Ph.D. degree in mechanical engineering in 2002 from the Ecole Centrale Nantes (France). Since 2005, he has held the post of Assistant Professor at the University of Brest, in France. He was awarded an accreditation to supervise research (HDR) in 2016.

Stéphane's research topics include the mechanical behavior of materials, assemblies and structures (experimental characterization and modeling).

Bernard Multon received his M.S. degree in electrical engineering from the Ecole Normale Supérieure de Cachan (France) in 1981, followed by an "Agrégation" credential in Electrical Engineering in 1982 and then a Ph.D. from the University of Paris 6 in 1985. Since 1995, he has held the post of Full Professor at ENS Rennes teaching in the Mechatronics Department.

His research is conducted with the SETE team of the SATIE-CNRS Laboratory and pertains to the sizing of electromechanical chains and the management of renewable electrical energy conversion systems throughout their life cycle.

Hamid Ben Ahmed received his Ph.D. degree and HDR accreditation in 1994 and 2006, respectively. From 1994 to 1997, he worked as a Research Associate in the SATIE Laboratory. Since 1997, Hamid has held the post of Assistant Professor in the Mechatronic Department at ENS Cachan (France), which went on to become ENS Rennes.

His research interests include the design, modeling and optimization of novel topologies for electromagnetic actuators and generators, as well as the optimization of renewable energy systems. 